\title{
Optimization of orders in multichannel fractional Fourier-domain filtering circuits and its application to the synthesis of mutual-intensity distributions
}

\author{
İmam Şamil Yetik, Mehmet Alper Kutay, and Haldun Memduh Ozaktas
}

\begin{abstract}
Owing to the nonlinear nature of the problem, the transform orders in fractional Fourier-domain filtering configurations have usually not been optimized but chosen uniformly. We discuss the optimization of these orders for multi-channel-filtering configurations by first finding the optimal filter coefficients for a larger number of uniformly chosen orders, and then maintaining the most important ones. The method is illustrated with the problem of synthesizing desired mutual-intensity distributions. The method we propose allows those fractional Fourier domains, which add little benefit to the filtering process but increase the overall cost, to be pruned, so that comparable performance can be attained with less cost, or higher performance can be obtained with the same cost. The method we propose is more likely to be useful when confronted with low-cost rather than high-performance applications, because larger improvements are obtained when the use of a smaller number of filters is desired. (C) 2002 Optical Society of America
\end{abstract}

OCIS codes: $\quad 070.6560,070.2590$.

\section{Introduction}

The fractional Fourier transform has found many applications in optics and signal processing. ${ }^{1-19}$ A comprehensive exposition to the subject and an extensive bibliography may be found in Ref. 20. The $a$ th order fractional Fourier-transform operation corresponds to the ath power of the ordinary Fouriertransform operation. If we denote the ordinary Fourier-transform operator by $\mathscr{F}$, then the ath-order fractional Fourier-transform operator is denoted by $\mathscr{F}^{\mathrm{a}}$. Standard eigenvalue methods for finding a function $G(\mathscr{A})$ of a linear operator $\mathscr{A}$ can be employed to obtain an explicit formula for the transform. If the eigenvalue equation of $\mathscr{A}$ is $\mathscr{A} \psi_{n}(u)=\lambda_{n} \psi_{n}(u)$, we define $G(\mathscr{A})$ through the eigenvalue equation $G(\mathscr{A}) \psi_{n}(u)=G\left(\lambda_{n}\right) \psi_{n}(u)$. The eigenvalue equation of the Fourier transform is $\mathscr{F}_{\psi_{n}}(u)=\exp (-i n \pi / 2) \psi_{n}(u)$,

İ. Ş. Yetik, is with the University of Illinois at Chicago, Department of Electrical and Computer Engineering, Chicago, Illinois. M. A. Kutay is with TÜBITAK-UEKAE (Scientific and Technical Research Council of Turkey-National Research Institute of Electronics and Cryptology) TR-06100 Kavaklidere, Ankara, Turkey. H. M. Ozaktas is with Bilkent University, Department of Electrical Engineering, TR-06533 Bilkent, Ankara, Turkey.

Received 6 March 2001; revised manuscript received 11 January 2002.

0003-6935/02/204078-07\$15.00/0

(C) 2002 Optical Society of America where $\psi_{n}(u), n=0,1,2, \ldots$ are the set of HermiteGaussian functions: $2^{1 / 4}\left(2^{n} n !\right)^{-1 / 2} H_{n}(\sqrt{2 \pi} u)$ $\exp \left(-\pi u^{2}\right)$, where $H_{n}(u)$ are the standard Hermite polynomials. Now, using the above approach, the fractional Fourier transform can be defined in terms of the eigenvalue equation $\mathscr{F}^{\mathrm{a}} \psi_{n}(u)=[\exp (-i n \pi /$ $2]^{a} \psi_{n}(u)$. We choose to resolve the ambiguity in the $a$ th power function as $[\exp (-i n \pi / 2)]^{a}=\exp (-i a n \pi /$ 2 ). To find the fractional Fourier transform of an arbitrary square-integrable function $f(u)$ we first expand it in terms of the complete orthonormal set of functions $\psi_{n}(u)$ and then apply the above eigenvalue equation to each term of the expansion. After rearranging the terms and using a standard identity for Hermite polynomials [Ref. 20, table 2.8.9], it is possible to show that the ath-order fractional Fourier transform $f_{\alpha}(u) \equiv \mathscr{F}^{\mathrm{a}} \mathrm{f}(u)$ of the original function is given by

$$
\begin{aligned}
f_{a}(u)= & {\left[1-i \cot \left(\frac{a \pi}{2}\right)\right]^{1 / 2} \int \exp \left\{i \pi \left[\cot \left(\frac{a \pi}{2}\right) u^{2}\right.\right.} \\
& \left.\left.-2 \csc \left(\frac{a \pi}{2}\right) u u^{\prime}+\cot \left(\frac{a \pi}{2}\right) u^{\prime 2}\right]\right\} f\left(u^{\prime}\right) \mathrm{d} u^{\prime} .
\end{aligned}
$$

The zeroth-order fractional Fourier transform of a function is the function itself and the first-order 
transform is equal to the ordinary Fourier transform. Positive and negative integer values of $a$ simply correspond to the repeated application of the ordinary forward and inverse Fourier transforms respectively. The fractional Fourier-transform operator satisfies the index additivity: $\mathscr{F}^{a_{2}} \mathscr{F}^{a_{1}}=\mathscr{F}^{a_{2}+a_{1}}$. The operator $\mathscr{F}^{\mathrm{a}}$ is periodic in $a$ with period 4 because $\mathscr{F}^{2}$ equals the parity operator, which maps $f(u)$ to $f(-u)$ and $\mathscr{F}^{4}$ equals the identity operator.

An important concept in Fourier analysis is the Fourier (or frequency) domain. This domain is understood to be a space where the Fourier-transform representation of $f(u)$ lives. The space-frequency plane (also known as phase space) is the plane spanned by the space $(u)$ and spatial frequency $(\mu)$ coordinates [Fig. 1(d)]. The horizontal axis is the space domain where $f(u)$ lives. The vertical axis is the frequency or Fourier domain where the Fourier transform $F(\mu)$ lives. In general, oblique axes $u_{a}$ making angle $\alpha=a \pi / 2$ with the $u$ axis are the $a$ thorder fractional Fourier domains, where the athorder fractional Fourier transforms $f_{a}\left(u_{a}\right)$ live. This notion is supported by the fact that fractional Fourier transformation corresponds to rotation of certain space-frequency distributions, such as the Wigner distribution, and that the integral projection of the Wigner distribution on the $u_{a}$ axis yields the $a$ thorder fractional Fourier energy density. ${ }^{20-25}$

The ath-order discrete fractional Fourier transform $\mathbf{f}_{a}$ of a given $N \times 1$ vector $\mathbf{f}$ is found as $\mathbf{f}_{a}=\mathbf{F}^{a} \mathbf{f}$, where $\mathbf{F}^{a}$ is the $N \times N$ discrete fractional Fouriertransform matrix, ${ }^{26}$ which is essentially the ath power of the ordinary discrete Fourier-transform matrix F. If the discrete vectors represent the samples of their continuous counterparts and if $N$ is chosen equal to or greater than the space-bandwidth product of $f(u)$, the discrete fractional transform approximates the continuous fractional transform in the same way as the ordinary discrete transform approximates the ordinary continuous transform.

\section{Fractional Fourier-Domain Filtering}

Space- and frequency-domain filtering are special cases of fractional Fourier-domain filtering (Figs. 1(a), 1(b), 1(c)]. ${ }^{24,27}$ Fractional Fourier-domain filtering consists of (i) taking the fractional Fourier transform of the input signal, (ii) multiplication with a filter function, and (iii) taking the inverse fractional Fourier transform of the result. The fractional version of the optimal Wiener filtering problem has been studied in detail. 27,28 Fractional Fourier-domain filtering has been further generalized to multistage and multichannel filtering [Figs. 1(e) and 1(f)]. In multistage filtering ${ }^{29-33}$ the input is first transformed into the $a_{1}$ th domain, where it is multiplied by a filter $\mathbf{h}_{1}$. The result is then transformed back into the original domain. This process is repeated $M$ times. Denoting the diagonal matrix corresponding to multiplication by the $k$ th filter $\mathbf{h}_{k}$ by $\mathbf{A}_{k}$, we can write the (a)

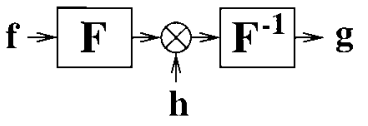

(c)

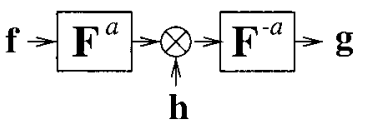

(b)

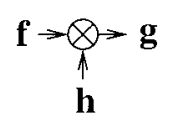

(d)

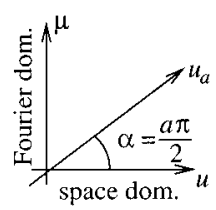

(e)

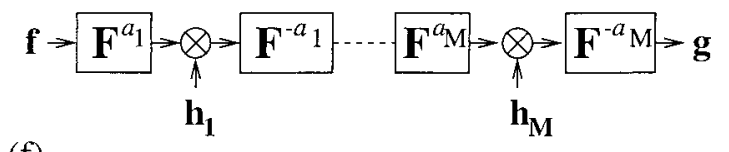

(f)

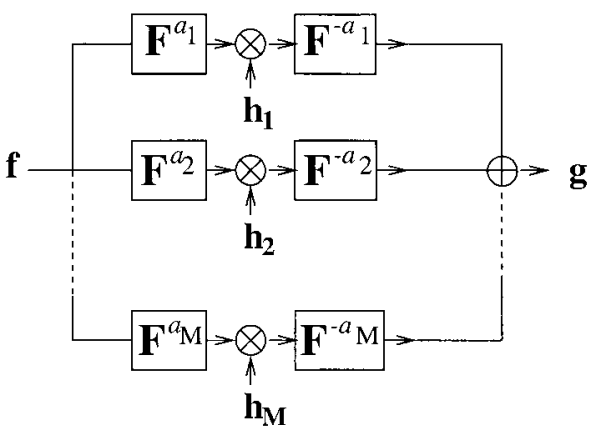

Fig. 1. (a) Fourier-domain filtering. (b) Space-domain filtering. (c) ath-order fractional Fourier domain filtering. (d) Fractional Fourier domains. (e) Multistage filtering. (f) Multichannel filtering.

following expression for the overall effect of the multistage filtering configuration:

$$
\mathbf{T}_{\mathrm{ms}}=\mathbf{F}^{-a_{M}} \Lambda_{M} \cdots \mathbf{F}^{a_{2}-a_{1}} \Lambda_{1} \mathbf{F}^{a_{1}},
$$

where $\mathbf{T}_{\mathrm{ms}}$ is a matrix representing the overall multi-stage-filtering configuration and $\mathbf{F}^{a_{k}}$ is the $a_{k}$ th-order discrete fractional Fourier-transform matrix. Multi-channel-filtering configurations ${ }^{31-35}$ consist of $M$ single-stage blocks in parallel. For each channel $k$, the input is transformed to the $a_{k}$ th domain, multiplied by a filter $\mathbf{h}_{k}$ and then transformed back. We can write the following expression for the overall effect of the multi-channel-filtering configuration:

$$
\mathbf{T}_{\mathrm{mc}}=\sum_{k=1}^{M} \mathbf{F}^{-a_{k}} \Lambda_{k} \mathbf{F}^{a_{k}},
$$

where $\mathbf{T}_{\mathrm{mc}}$ is a matrix representing the overall multichannel-filtering configuration. It is possible to further generalize these filtering configurations by use of parallel and series arrangements together; such systems have been called generalized filtering circuits. 36

Fractional Fourier transform-based filtering circuits have found applications in many areas including optical and digital signal and image 


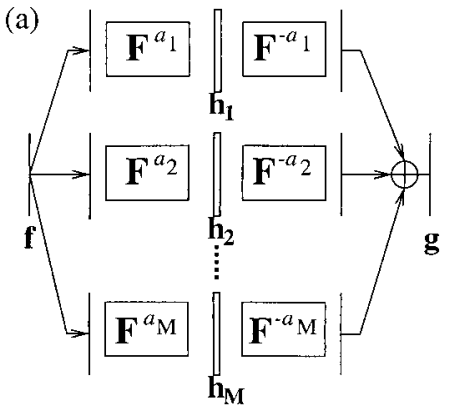

(c)

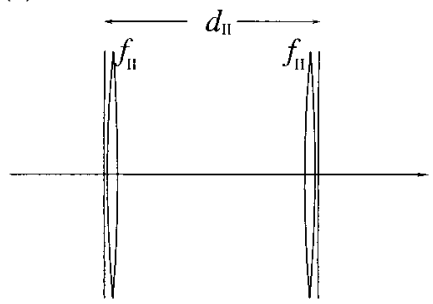

(b)

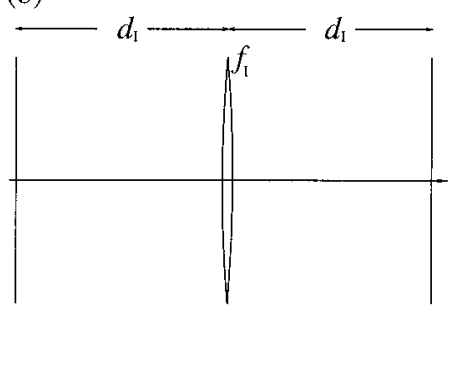

(d)

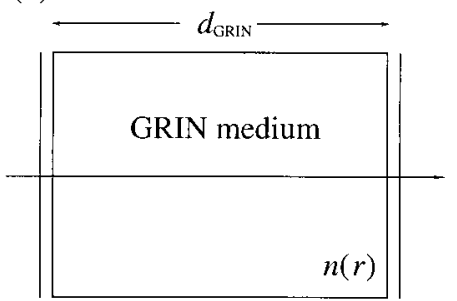

Fig. 2. (a) The multichannel configuration consists of $k=1,2, \ldots, M$ parallel channels, each consisting of a fractional Fourier-transform stage $\mathbf{F}^{a_{k}}$ followed by a spatial filter $\mathbf{h}_{k}$ followed by another fractional Fourier-transform stage of order $-a_{k}$. (b), (c) and (d) show three alternative optical implementations of the fractional Fourier-transform stages appearing in (a) (b) Canonical implementation type I. (c) Canonical implementation type II. (d) Quadratic graded-index (GRIN) medium implementation. While the focal lengths of the lenses and their separations are shown in (b) and (c) the radial-refractive-index distribution is given by $n^{2}(r)=n_{0}^{2}\left[1-(r / \chi)^{2}\right]$.

restoration, signal and system synthesis, synthesis of mutual-intensity distributions, and fast implementation of shift-variant linear systems (Ref. 20, Chap. 10).

The problem of finding the optimal filter coefficients, given the transform orders, was solved. ${ }^{27,30,33}$ Given a matrix $\mathbf{H}$ that represents a system one wishes to synthesize, one seeks the filter coefficients such that the resulting matrices $\mathbf{T}_{\mathrm{ms}}$ or $\mathbf{T}_{\mathrm{mc}}$ are as close as possible to $\mathbf{H}$ according to some specified criteria, such as mean square error. Until now the transform orders have usually been chosen uniformly; the problem of optimizing the orders has not yet been addressed. In this paper we show how one can optimize over the orders for multichannel filtering by first finding the optimal filter coefficients for a larger number of uniformly chosen orders and then maintaining the most important ones.

Fractional Fourier-transform-based filtering configurations have been used for approximating linear space-variant systems, represented by some matrix H. ${ }^{30-32,34}$ It was shown that for many such systems encountered in various applications, it is possible to approximate the system $\mathbf{H}$ with a multistage or multichannel configuration $\mathbf{T}_{\mathrm{ms}}$ or $\mathbf{T}_{\mathrm{mc}}$ with acceptable mean square error, by using a small or moderate number $M$ of stages or channels. Because the cost of implementing the fractional Fourier transform (optically or digitally) is similar to the cost of implementing the ordinary Fourier transform, this leads to an efficient implementation of the space-variant system in question. For digi- tal systems, the cost is of the order of $M N \log N$, which should be compared to a cost of the order of $N^{2}$ for direct implementation of linear systems. (Here $N$ is the length of the discrete signal vectors that should be at least as large as the spacebandwidth product of the continuous signals.)

In the multichannel case it is possible to analytically find the optimal filter coefficients, provided that the transform orders are given. ${ }^{33}$ In practice, however, an iterative method is preferred. In the multistage case it is not possible to find analytic solutions, so an iterative method must be used to begin with. ${ }^{30}$

In this paper we concentrate on the multi-channelfiltering case and consider the improvement of optimizing over the $M$ orders in addition to the filter coefficients. We first find the optimal filter coefficients for a larger number $P$ of uniformly chosen orders and then maintain the most important ones. More specifically, we start with $P$ uniformly chosen orders, where $P$ is several times the number of orders $M$ we are eventually going to use. Then the $M$ orders resulting in filters with the highest energies are chosen, and the other $P-M$ branches of the multichannel configuration are eliminated. Finally, with the $M$ orders thus chosen, we reoptimize the filter coefficients.

Figure 2 shows how the fractional Fouriertransform stages of the multichannel configuration can be optically implemented. ${ }^{21,37-39}$ Either of the alternative implementations shown [in Figs. 2(b), $2(\mathrm{c})$, or $2(\mathrm{~d})$ ] can be used for the fractional Fourier blocks appearing in Fig. 2(a). For a fractional trans- 
form of order $a$, the parameters of the configurations should be chosen as follows:

Fig. 2(b): $\quad \mathrm{d}_{I}=\frac{s^{2}}{\lambda} \tan (a \pi / 4), \quad f_{I}=\frac{s^{2}}{\lambda} \csc \left(\frac{a \pi}{2}\right)$,

Fig. 2(c): $\quad \mathrm{d}_{I I}=\frac{s^{2}}{\lambda} \sin (a \pi / 2), \quad f_{I I}=\frac{s^{2}}{\lambda} \cot \left(\frac{a \pi}{4}\right)$,

Fig. 2(d): $\mathrm{d}_{\text {GRIN }}=\frac{s^{2}}{\lambda}(a \pi / 2), \quad \chi G R I N=\frac{s^{2}}{\lambda}$,

where $\lambda$ is the wavelength and $s$ is a suitably chosen scale parameter with the dimension of length. These configurations will map a function $f(x / s)$ at their input to a function $f_{a}(x / s)$ at their output, where $x$ is measured in meters.

\section{Synthesis of Mutual-Intensity Distributions}

To illustrate our approach, we will consider the problem of synthesizing light with a desired mutual intensity. Here we wish to synthesize a system $\mathbf{H}$ such that, when light of a given mutual intensity is present at the input, light whose mutual intensity is as close as possible to the given specification is obtained at the output. Choosing to work with onedimensional signals taking dimensionless variables for simplicity, we let $f(u)$ and $g(u)$ denote the input and output optical fields, and $R_{f}\left(u_{1}, u_{2}\right)$ and $R_{g}\left(u_{1}, u_{2}\right)$ denote the input and output mutual intensities. If $f(u)$ and $g(u)$ are the input and output of a system characterized by a kernel $H\left(u, u^{\prime}\right)$ such that $g(u)=f$ $H\left(u, u^{\prime}\right) f\left(u^{\prime}\right) \mathrm{d} u^{\prime}$, then the input and output mutual intensities are related by

$$
\begin{aligned}
R_{g}\left(u_{1}, u_{2}\right)= & \iint R_{f}\left(u_{1}{ }^{\prime}, u_{2}{ }^{\prime}\right) H\left(u_{1}, u_{1}{ }^{\prime}\right) \\
& \times H^{*}\left(u_{2}, u_{2}{ }^{\prime}\right) \mathrm{d} u_{1}{ }^{\prime} \mathrm{d} u_{2}{ }^{\prime},
\end{aligned}
$$

where $H^{*}$ denotes the complex conjugate of $H$. The sampled, discrete version of the optical fields will be represented by column vectors $\mathbf{f}$ and $\mathbf{g}$ and the mutual intensity functions will be represented by matrices $\mathbf{R}_{f}$ and $\mathbf{R}_{g}$. Then, we have $\mathbf{g}=\mathbf{H f}$, where $\mathbf{H}$ is the discrete form of the system kernel and the double integral relationship above assumes the following matrix form:

$$
\mathbf{R}_{\mathrm{g}}=\mathbf{H} \mathbf{R}_{f} \mathbf{H}^{\dagger},
$$

where $\mathbf{H}^{\dagger}$ is the Hermitian conjugate of $\mathbf{H}$. Equation (8) is quadratic in $\mathbf{H}$. We are going to employ an equivalent representation that is linear. Because mutual intensity matrices $\mathbf{R}$ are Hermitian and positive semi-definite, it is possible to diagonalize them as

$$
\mathbf{R}=\mathbf{U D U}^{\dagger},
$$

where $\mathbf{D}$ is a diagonal matrix whose elements are the real eigenvalues, and $\mathbf{U}$ is a matrix whose columns constitute the set of orthonormal eigenvectors of $\mathbf{R}$ so that $\mathbf{U}^{\dagger} \mathbf{U}=\mathbf{I}$, where $\mathbf{I}$ is the identity matrix. Letting $\mathbf{D}^{1 / 2}$ denote the diagonal matrix whose elements are the positive square roots of the elements of $\mathbf{D}$, we substitute $\mathbf{D}^{1 / 2} \mathbf{U}^{\dagger} \mathbf{U} \mathbf{D}^{1 / 2}$ for $\mathbf{D}$ in the above equation:

$$
\mathbf{R}=\mathbf{U D}^{1 / 2} \mathbf{U}^{\dagger} \mathbf{U D}^{1 / 2} \mathbf{U}^{\dagger}
$$

Using this expansion for both $\mathbf{R}_{g}$ and $\mathbf{R}_{f}$, we can write

$$
\begin{aligned}
\mathbf{R}_{g} & =\tilde{\mathbf{R}}_{g} \tilde{\mathbf{R}}_{g}^{\dagger}=\tilde{\mathbf{R}}_{g} \tilde{\mathbf{R}}_{g}=\tilde{\mathbf{R}}_{g}{ }^{2}, \\
\mathbf{R}_{f} & =\tilde{\mathbf{R}}_{f} \tilde{\mathbf{R}}_{f}^{\dagger}=\tilde{\mathbf{R}}_{f} \tilde{\mathbf{R}}_{f}=\tilde{\mathbf{R}}_{f}^{2},
\end{aligned}
$$

where

$$
\begin{aligned}
\tilde{\mathbf{R}}_{g} & =\tilde{\mathbf{R}}_{g}^{\dagger}=\mathbf{U D}^{1 / 2} \mathbf{U}^{\dagger}, \\
\tilde{\mathbf{R}}_{f} & =\tilde{\mathbf{R}}_{f}^{\dagger}=\mathbf{U D}^{1 / 2} \mathbf{U}^{\dagger} .
\end{aligned}
$$

Substituting Eq. (11) into Eq. (8) we obtain the following:

$$
\tilde{\mathbf{R}}_{g} \tilde{\mathbf{R}}_{g}^{\dagger}=\mathbf{H} \tilde{\mathbf{R}}_{f} \tilde{\mathbf{R}}_{f}^{\dagger} \mathbf{H}^{\dagger} .
$$

One way of satisfying the above equation is to ensure that

$$
\tilde{\mathbf{R}}_{g}=\mathbf{H} \tilde{\mathbf{R}}_{f},
$$

or

$$
\mathbf{H}=\tilde{\mathbf{R}}_{g} \tilde{\mathbf{R}}_{f}^{-1} .
$$

In our numerical examples, we are going to consider the input light source to be incoherent. Assuming this source extends uniformly from $-r_{0}$ to $r_{0}$, its mutual intensity can be written as

$$
\mathbf{R}_{f}\left(u_{1}, u_{2}\right)=\delta\left(u_{1}-u_{2}\right) \operatorname{rect}\left(\frac{u_{1}}{2 r_{0}}\right) .
$$

When discretized, the corresponding matrix $\mathbf{R}_{f}$ (and its square root $\tilde{\mathbf{R}}_{f}$ ) is equal to the identity I provided that $r_{0}$ is larger than the interval over which we sample. Therefore the matrix $\mathbf{H}$ we wish to approximate is simply equal to $\tilde{\mathbf{R}}_{g}$.

As a first example, we wish to synthesize a Gaussian-Schell-model beam with mutual intensity:

$$
R_{g}\left(u_{1}, u_{2}\right)=\exp \left[-\frac{\left(u_{1}-u_{2}\right)^{2}}{2 r_{1}^{2}}\right] \exp \left(-\frac{u_{1}^{2}+u_{2}^{2}}{4{r_{2}}^{2}}\right) \text {. }
$$

(In our examples $r_{1}=5$ and $r_{2}=10$.) When we synthesize the filter $\mathbf{H}$ corresponding to this mutual intensity using the multichannel configuration with $M=3$ filters $\left(a_{1}=1 / 3, a_{2}=2 / 3, a_{3}=1\right)$, the normalized error turns out to be $15.42 \%$. Using the proposed method of optimizing the orders with $P=$ 12 , we find that the optimal orders are $a_{1}=2 / 12$, $a_{2}=5 / 12, a_{3}=10 / 12$, and the normalized error using these orders becomes $12.64 \%$. When we synthesize the same $\mathbf{H}$ with $M=2$ filters $\left(a_{1}=1 / 2, a_{2}=\right.$ 1 ), the normalized error is $22.36 \%$. Optimizing the 
(a)

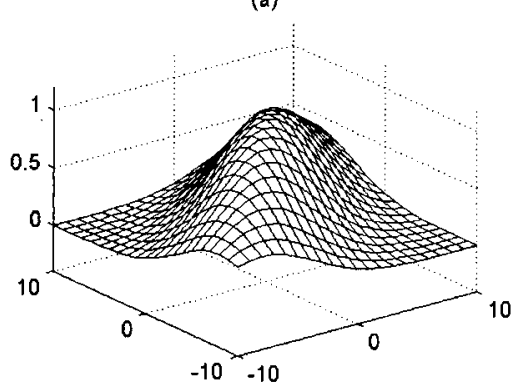

(c)

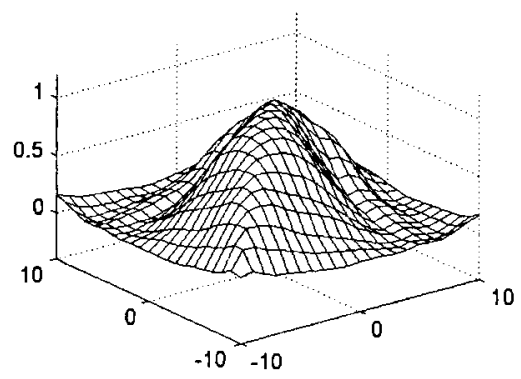

(b)

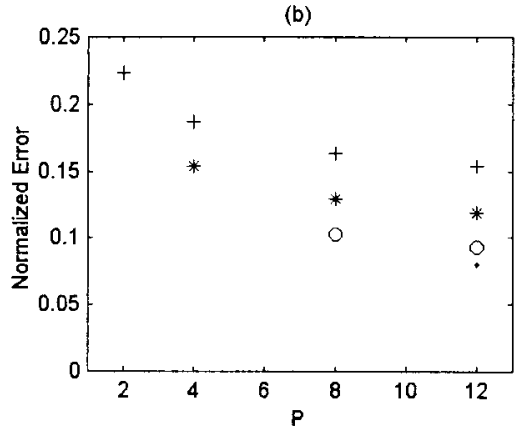

(d)

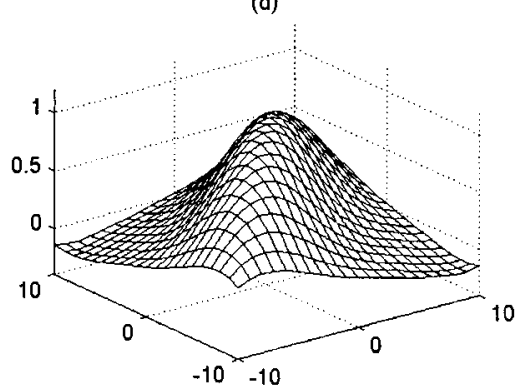

Fig. 3. (a) Desired Gaussian-Schell-model mutual intensity profile. (b) Normalized error versus $P$ for different values of $M$. We show $M=2, P=2,4,8,12$ by crosses; $M=4, P=4,8,12$ by asterisks $M=8, P=8,12$ by open circles, and $M=12, P=12$ by dots. $\quad(\mathrm{c})$ Synthesized profile using uniform orders $(M=2)$. (d) Synthesized profile using optimized orders $(M=2, P=8)$.

orders with $P=8$, we find that the optimal orders are $a_{1}=2 / 8, a_{2}=6 / 8$, and the normalized error using these orders is $16.36 \%$. Further simulations have been undertaken for other values of $M$ and $P$ and the resulting errors are plotted in Fig. 3(b). Figure 3(a) shows the desired mutual intensity, Fig. 3(c) shows the synthesized mutual intensity for $M=2$ without optimization of orders, and Fig. 3(d) shows the synthesized mutual intensity for $M=2$ with optimization of orders with $P=8$.

As a second example we consider the synthesis, as closely as possible, of a mutual-intensity profile specified as

$$
R_{g}\left(u_{1}, u_{2}\right)=\operatorname{rect}\left(\frac{\left|u_{1}-u_{2}\right|}{2 r_{1}}\right) \operatorname{rect}\left(\frac{u_{1}}{2 r_{2}}\right) \operatorname{rect}\left(\frac{u_{2}}{2 r_{2}}\right) \text {, }
$$

where $r_{2}>r_{1}$. This amounts to specifying the amplitude of light at two points to be fully correlated when the distance between those points is less than $r_{1}$, and totally uncorrelated otherwise. Because the rectangle function does not represent a physically realizable mutual-intensity function (it is not positive semi-definite), its negative eigenvalues will be replaced by zero in obtaining its square root representation. This amounts to replacing the rectangle function with the closest positive semi-definite function. When we synthesize the filter $\mathbf{H}$ corresponding to this mutual intensity using the multichannel configuration with $M=3$ filters $\left(a_{1}=1 / 3, a_{2}=2 / 3\right.$, $a_{3}=1$ ), the normalized error is $15.35 \%$. Using the proposed method of optimizing the orders with $P=12$, we find that the optimal orders are $a_{1}=2 / 12$, $a_{2}=6 / 12, a_{3}=10 / 12$, and the normalized error using these orders is $12.3 \%$. When we synthesize the same $\mathbf{H}$ with $M=2$ filters $\left(a_{1}=1 / 2, a_{2}=1\right)$, the normalized error is $22.64 \%$. Optimizing the orders with $P=8$, we find that the optimal orders are $a_{1}=2 / 8, a_{2}=6 / 8$, and the normalized error using these orders is $15.45 \%$. Once again, further simulations were undertaken for other values of $M$ and $P$ and are plotted in Fig. 4(b). Figure 4(a) shows the desired mutual intensity, Fig. 4(c) shows the synhthesized mutual intensity for $M=2$ without optimization of orders, and Fig. 4(d) shows the synthesized mutual intensity for $M=2$ and optimization of orders with $P=8$.

A number of conclusions can be drawn by examining the numerical results. First, optimization of the orders is capable of offering tangible improvements compared to choosing the orders uniformly. We also observe that beyond a certain value of $P$, further increases in this parameter do not offer further reductions in the error (the benefits of optimizing over the orders is saturated). This is because further increasing $P$ merely allows further refinements and fine tuning in choosing the optimal orders that which have a diminishing return once one is already close to the optimal orders. Also, we can see that improvements coming from optimization of the orders are greater when $M$ is smaller but less when $M$ is larger. This is because when $M$ is large to begin with, it is already possible to concentrate the filtering action in those domains that are optimal. This of course means that the other domains add cost to the system implementation with little benefit, and the method 

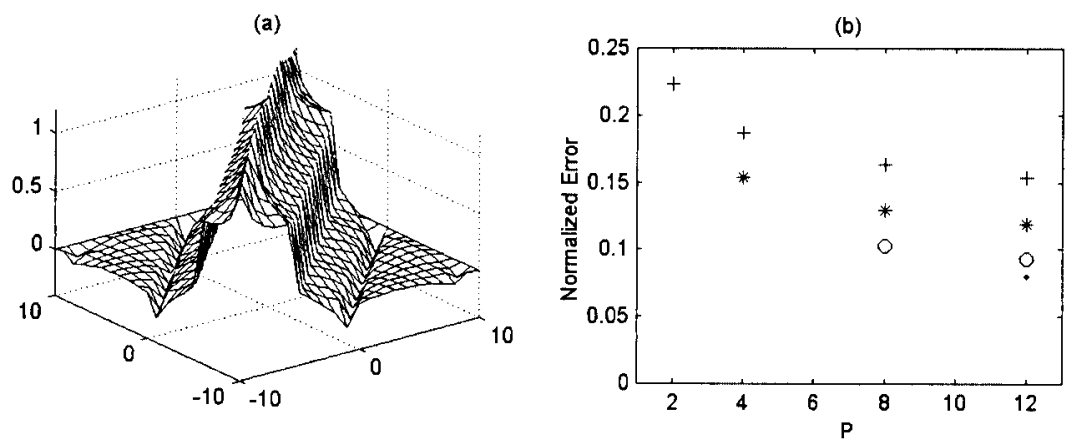

(c)
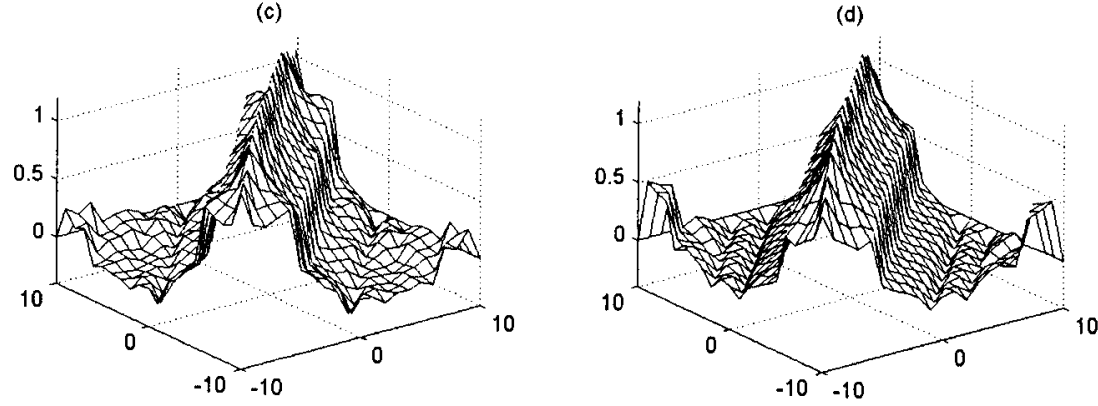

Fig. 4. (a) Closest positive semi-definite approximation to the desired rectangular mutual intensity profile. (b) Normalized error versus $P$ for different values of $M$. We show $M=2, P=2,4,8,12$ by pluses; $M=4, P=4,8,12$ by asterisks $M=8, P=8,12$ by open circles, and $M=12, P=12$ by dots. (c) Synthesized profile using uniform orders $(M=2)$. (d) Synthesized profile using optimized orders $(M=$ $2, P=8)$.

we propose is useful precisely because it allows these low benefit domains to be pruned.

\section{Conclusion}

In conclusion, we have presented a simple and effective way of optimizing the orders in fractional Fourier-domain-based multi-channel-filtering configurations. Until now, the orders had mostly been chosen uniformly because there was no simple way of solving the nonlinear problem of optimizing over the orders. The method we propose is more likely to be useful when confronted with low-cost, rather than high-accuracy applications, because larger improvements are obtained when the use of a smaller number of filters is desired. Future work might include extending the method to the multistage case, which poses a number of challenges, and to more general filtering circuits. Generalizing the procedure to the optimization of the parameters of linear canonical transform-based-filtering systems, ${ }^{40}$ which are even more general than fractional Fourier-transformbased systems, would have the potential to offer further improvements.

\section{References}

1. L. B. Almeida, "The fractional Fourier transform and timefrequency representations," IEEE Trans Signal Process. 42, 3084-3091 (1994).

2. O. Akay and G. F. Boudreaux-Bartels, "Unitary and Hermitian fractional operators and their relation to the fractional Fourier transform," IEEE Signal Process. Lett. 5, 312-314 (1998).

3. T. Alieva, V. Lopez, F. Agullo-Lopez, and L. B. Almeida, "The fractional Fourier transform in optical propagation problems," J. Mod. Opt. 41, 1037-1044 (1994).
4. İ. Ş. Yetik, H. M. Ozaktas, B. Barshan, and L. Onural, "Perspective projections in the space-frequency plane and fractional Fourier transforms," J. Opt. Soc. Am. A 17, 2382-2390 (2000).

5. L. M. Bernardo and O. D. D. Soares, "Fractional Fourier transforms and imaging," J. Opt. Soc. Am. A 11, 2622-2626 (1994).

6. W. X. Cong, N. X. Chen, and B. Y. Gu, "Recursive algorithm for phase retrieval in the fractional Fourier transform domain," Appl. Opt. 37, 6906-6910 (1998).

7. D. Dragoman and M. Dragoman, "Near and far field optical beam characterization using the fractional Fourier tansform," Opt. Commun. 141, 5-9 (1997).

8. M. F. Erden, H. M. Ozaktas, and D. Mendlovic, "Propagation of mutual intensity expressed in terms of the fractional Fourier transform," J. Opt. Soc. Am. A 13, 1068-1071 (1996).

9. M. F. Erden, H. M. Ozaktas, and D. Mendlovic, "Synthesis of mutual intensity distributions using the fractional Fourier transform," Optics Commun. 125, 288-301 (1996).

10. J. García, R. G. Dorsch, A. W. Lohmann, C. Ferreira, and Z. Zalevsky, "Flexible optical implementation of fractional Fourier transform processors. Applications to correlation and filtering," Opt. Commun. 133, 393-400 (1997).

11. S. Granieri, R. Arizaga, and E. E. Sicre, "Optical correlation based on the fractional Fourier transform," Appl. Opt. 36, 6636-6645 (1997).

12. J. Hua, L. Liu, and G. Li, "Scaled fractional Fourier transform and its optical implementation," Appl. Opt. 36, 8490-8492 (1997).

13. C. J. Kuo and Y. Luo, "Generalized joint fractional Fourier transform correlators: a compact approach,” Appl. Opt. 37, 8270-8276 (1998).

14. S. Liu, J. Xu, Y. Zhang, L. Chen, and C. Li, "General optical implementation of fractional Fourier transforms," Opt. Lett. 20, 1053-1055 (1995).

15. A. W. Lohmann, Z. Zalevsky, and D. Mendlovic, "Synthesis of 
pattern recognition filters for fractional Fourier processing," Opt. Commun., 128, 199-204 (1996).

16. D. Mendlovic, Z. Zalevsky, and H. M. Ozaktas, "Applications of the fractional Fourier transform to optical pattern recognition," in Optical Pattern Recognition, (Cambridge University Press, Cambridge, 1998) Chap. 4, pp. 89-125.

17. P. Pellat-Finet, "Fresnel diffraction and the fractional-order Fourier transform," Opt. Lett. 19, 1388-1390 (1994).

18. Z. Zalevsky, D. Medlovic, and H. M. Ozaktas, "Energetic efficient synthesis of general mutual intensity distribution," J. Opt. Soc. A 2, 83-87 (2000).

19. Y. Zhang and B.-Y. Gu, "Rotation-invariant and controllable space-variant optical correlation,” Appl. Opt. 37, 6256-6261 (1998).

20. H. M. Ozaktas, Z. Zalevsky, and M. A. Kutay, The Fractional Fourier Transform with Applications in Optics and Signal Processing, (John Wiley \& Sons, New York, 2001).

21. A. W. Lohmann, "Image rotation, Wigner rotation, and the fractional order Fourier transform," J. Opt. Soc. Am. A 10, 2181-2186 (1993).

22. A. W. Lohmann and B. H. Soffer, "Relationships between the Radon-Wigner and fractional Fourier transforms," J. Opt. Soc. Am. A 11, 1798-1801 (1994).

23. D. Mustard, "The fractional Fourier transform and the Wigner distribution,” J Aust. Math. Soc. B 38, 209-219 (1996).

24. H. M. Ozaktas, B. Barshan, D. Mendlovic, and L. Onural, "Convolution, filtering, and multiplexing in fractional Fourier domains and their relation to chirp and wavelet transforms," J. Opt. Soc. Am. A 11, 547-559 (1994).

25. H. M. Ozaktas, B. Barshan, and D. Mendlovic, "Convolution and filtering in fractional Fourier domains," Opt. Rev. 1, 15-16 (1994).

26. C. Candan, M. A. Kutay, and H. M. Ozaktas, "The discrete fractional Fourier transform,” IEEE Trans. Signal Process. 48, 1329-1337 (2000).

27. M. A. Kutay, H. M. Ozaktas, O. Arikan, and L. Onural, "Optimal filtering in fractional Fourier domains," IEEE Trans. Signal Process. 45, 1129-1143 (1997).

28. Z. Zalevsky and D. Mendlovic, "Fractional Wiener filter," Appl. Opt. 35, 3930-3936 (1996).

29. M. F. Erden, M. A. Kutay, and H. M. Ozaktas, "Repeated filtering in consecutive fractional Fourier domains and its application to signal restoration," IEEE Trans. Signal Process. 47, 1458-1462 (1999).
30. M. F. Erden and H. M. Ozaktas, "Synthesis of general linear systems with repeated filtering in consecutive fractional Fourier domains," J. Opt. Soc. Am. A 15, 1647-1657 (1998).

31. M. A. Kutay, M. F. Erden, H. M. Ozaktas, O. Arikan, Ö. Güleryüz, and C. Candan, "Space-bandwidth-efficient realizations of linear systems," Opt. Lett. 23, 1069-1071 (1998).

32. M. A. Kutay, M. F. Erden, H. M. Ozaktas, O. Arikan, C. Candan, and Ö. Güleryüz, "Cost-efficient approximation of linear systems with repeated and multi-channel filtering configurations," in Proceedings of the 1998 IEEE International Conference on Acoustics, Speech, and Signal Process. (IEEE, Piscataway, N. J., 1998) pp. 3433-3436.

33. M. A. Kutay, H. Özaktaş, M. F. Erden, H. M. Ozaktas, and O. Arikan, "Solution and cost analysis of general multi-channel and multi-stage filtering circuits," in Proceedings of the 1998 IEEE-SP International Symposium on Time-Frequency and Time-Scale Analysis (IEEE, Piscataway, N. J., 1998) pp. 481484.

34. M. A. Kutay, H. Özaktaş, H. M. Ozaktas, and O. Arikan, "The fractional Fourier domain decomposition," Signal Process. 77, 105-109 (1999).

35. İ. Ş. Yetik, M. A. Kutay, H. Özaktaş, and H. M. Ozaktas, "Continuous and discrete fractional Fourier domain decomposition, "in Proceedings of the 2000 IEEE International Conference on Acoustics, Speech, and Signal Processing, (IEEE, Piscataway, N. J., 2000) Vol. I, pp. 93-96.

36. H. M. Ozaktas, M. A. Kutay, and D. Mendlovic, "Introduction to the fractional Fourier transform and its applications" in P. W. Hawkes, ed., Advances in Imaging and Electron Physics, Vol. 106 (Academic Press, San Diego, Calif., 1999) Chap. 4, pp. 239-291.

37. D. Mendlovic and H. M. Ozaktas, "Fractional Fourier transforms and their optical implementation: I, J. Opt. Soc. Am. A 10, 1875-1881 (1993).

38. H. M. Ozaktas and D. Mendlovic "Fractional Fourier transforms and their optical implementation: II." J. Opt. Soc. Am. A 10, 2522-2531 (1993).

39. H. M. Ozaktas and D. Mendlovic, "Fractional Fourier optics," J. Opt. Soc. Am. A 12, 743-751 (1995).

40. B. Barshan, M. A. Kutay, and H. M. Ozaktas, "Optimal filtering with linear canonical transformations," Opt. Commun. 135, 32-36 (1997). 